\title{
ANÁLISE DOS DADOS DE PREVALENCIA DE CÁRIE DENTAL NA CIDADE DE BARRETOS, SP, BRASIL, DEPOIS DE DEZ ANOS DE FLUORETAÇÃO DA ÁGUA DE ABASTECIMENTO PUBLICO
}

Yvette Viegas*

Alfredo Reis Viegas*

\begin{abstract}
VIEGAS, Y, \& VIEGAS, A.R. Análise dos dados de prevalência de cárie dental na cidade de Barretos, SP, Brasil, depois de dez anos de fluoretação da água de abastecimento público. Rev. Saúde públ., S. Paulo, $19: 287-99,1985$.
\end{abstract}

RESUMO: Foram analisados os resultados dos dados de prevalência da cárie dental de crianças de 3 a 14 anos de idade e adultos jovens de 15 a 19 anos, da cidade de Barretos, SP, Brasil. Objetivou-se verificar as reduções de cárie dental encontradas em dentes primários e permanentes durante o período de dez anos de fluoretação da água de abastecimento público. A redução observada na prevalência de cárie para os dentes primários e permanentes foi similar à constatada em outros estudos, quer no Brasil ou no Exterior. No grupo etário de 7 a 10 anos, 50\% das crianças não tiveram nenhum dente permanente atacado pela cárie, e nas crianças de 3 a 5 anos, $51,6 \%$ não tiveram nenhum dente primário atacado pela cárie.

UNITERMOS: Fluoretação da água. Cárie dentăria, prevenção. Cárie dentária, prevalência.

Em junho de 1971, antes de ser iniciada a fluoretação da água de abastecimento público, no município de Barretos, SP, Brasil, foi feito o levantamento base da prevalência de cárie dental na população daquele município. $O$ objetivo era o de verificar a prevalência de cárie nesse ano nos dentes primários e permanentes para que se pudesse compará-la com dados de novos levantamentos que fossem feitos em anos futuros a fim de se avaliar a eficiência do método e, concomitantemente, demonstrar à população os benefícios decorrentes de sua aplicação. Assim sendo, em 1981, portanto dez anos depois de iniciada a fluoretação, novo levantamento foi realizado.

\section{METODOLOGIA}

Os examinadores, neste e no levantamento de 1971 , foram cirurgiões-dentistas de Barretos calibrados por docentes da Faculdade de Saúde Pública, autorés deste trabalho. Os dados levantados foram simples, objetivando: a) levantamentos que pudessem ser executados num curto período de tempo; b) que assegurassem confiança; e c) que permitissem aferir a prevalência de cárie por meio de índices simplificados e percentagens.

Foram utilizados quatro tipos de ficha, um para as crianças de 3 a 5 anos de idade, outro para as de 6 , um outro para as de 7 a 14, e um quarto tipo para os adultos jovens de 15 a 19 anos.

A ficha para as crianças de 3 a 5 possibilitava anotar os dados das crianças com dentes cariados, obturados, extraídos e hígidos; bem como os das crianças em relação ao número de dentes cariados e extraídos, ambos divididos em três categorias, zero, 1 a 4 e 5 e mais.

A ficha para as crianças de 6 anos, além dos dados contidos na ficha para

\footnotetext{
- Do Departamento de Prática de Saúde Pública da Faculdade de Saúde Pública da Universidade de São Paulo - Av. Dr. Arnaldo. 715 - 01255 - São Paulo, SP - Brasil.
} 
VIEGAS, Y. \& VIEGAS, A.R. Análise dos dados de prevalência de cárie dental na cidade de Barretos, SP, Brasil, depois de dez anos de fluoretação da água de abastecimento público. Rev. Saúde públ., S. Paulo, 19:287-99, 1985.

crianças de 3 a 5 anos, permitia ainda incluir o ataque de cárie do primeiro molar inferior direito.

A ficha para as crianças de 7 a 14 anos, além dos dados a ela pertinentes, possibilitava anotar $o$ ataque de cárie dos incisivos centrais superiores.

A ficha para os indivíduos de 15 a 19 anos de idade permitia anotar os dados referentes à necessidade ou ao uso de dentaduras e ao número de dentes cariados, divididos em três categorias: zero, 1 a 4 e 5 e mais; e ao número de dentes extraídos, divididos em seis categorias: zero, 1 a 4,5 a 10,11 a 16,17 a 22 e 23 a 28 . No levantamento de 1971 esta ficha foi utilizada para os indivíduos de 15 a 75 anos ou mais.

Dos dados obtidos no levantamento feito, calculou-se:

a) Nas crianças de 3 a 5 anos de idade:

- Percentagem de crianças com dentes hígidos, cariados, extraídos e obturados.

- Distribuição percentual de crianças segundo o número de dentes cariados e extraídos.

b) Nas crianças de 6 a 14 anos de idade:

- Percentagem de crianças com dentes hígidos, cariados, obturados e extraídos.

- Distribuição percentual de crianças segundo o número de dentes cariados e dentes extraídos.

- Número médio de dentes atacados pela cárie, estimado pelo índice de Viegas.

- Percentagem de crianças com primeiro molar inferior direito $e$ incisivos centrais superiores atacados pela cárie.

c) $\mathrm{Na}$ população de adultos jovens (15 a

19 anos de idade):

- Percentagem de indivíduos usando dentaduras.
- Distribuição percentual de indivíduos segundo o número de dentes cariados e dentes extraídos.

O exame para se obter os dados dos índices MID e/ou 2ICS (Viegas ${ }^{8}, 1969$ ) foi feito utilizando-se espelho e explorador; para os outros dados a técnica de exame foi visual, usando-se como elemento auxiliar, quando necessário, uma espátula de madeira. tes:

Os critérios adotados foram os seguin-

Dente hígido - quando não apresentar história de cárie.

Dente cariado - a) nas superfícies lisas, quando houver evidência de esmalte socavado, devendo haver uma cavidade definida na qual o explorador penetre; b) no caso de fissuras e fossetas, quando a ponta do explorador prender e desde que pelo menos uma das seguintes condições esteja presente: existe tecido cariado amolecido; há opacidade de esmalte ou mudança de coloração; c) nas superfícies proximais, quando a ponta do explorador prender, ficando retido quando se faz movimentos para oclusal e cervical.

Dente obturado - quando apresentar restauração com material permanente, tal como ouro, acolite, amálgama, cimento de silicato, resinas compostas, coroa total ou pivot.

Dente extraído - quando estiver ausente após a época normal de erupção (permanentes) ou antes da época normal de muda (primários).

Dentaduras - o indivíduo será incluído no cômputo dos que necessitam dentaduras (superior, inferior ou ambas), quando já tiver dentadura, bem como quando tiver todos os dentes extraídos num dos maxilares ou em ambos. 
VIEGAS, Y. \& VIEGAS, A.R. Análise dos dados de prevalência de cárie dental na cidade de Barretos, SP, Brasil, depois de dez anos de fluoretação da água de abastecimento público. Rev. Saúde públ., S. Paulo, 19:287-99, 1985.

No levantamento base de 1971 foram examinados habitantes de todas as idades, isto é, de 3 anos até 75 ou mais.

No levantamento de 1981 foram examinados apenas habitantes de 3 a 19 anos de idade, por serem os que já poderiam ter sido beneficiados pela adição de flúor à água de abastecimento após 10 anos.

A amostra foi constituída de indivíduos de 6 a 19 anos de idade que foram selecionados através de amostragem sistemática entre os que estavam matriculados nas escolas de ensino da rede estadual de Barretos; e os de 3 a 5 anos entre as crianças que frequientavam os parques infantis e escolas de pré-escolares.

$\mathrm{Na}$ Tabela 1 pode-se verificar o númeto de habitantes examinados em Barretos nas idades de 3 a 19 anos de idade, nos levantamentos realizados em 1971 e 1981.

TABELA 1

Número de crianças examinadas em Barretos, SP, segundo o sexo, idade, grupo etário e ano do levantamento (1971 e 1981)

\begin{tabular}{|c|c|c|c|c|c|c|}
\hline \multirow{2}{*}{ Idade } & \multicolumn{3}{|c|}{1971} & \multicolumn{3}{|c|}{1981} \\
\hline & Masculino & Feminino & Total & Masculino & Feminino & Total \\
\hline \multirow{8}{*}{$3-1$} & 71 & 52 & 123 & 60 & 56 & 116 \\
\hline & 78 & 74 & 152 & 72 & 59 & 131 \\
\hline & 90 & 95 & 185 & 74 & 127 & 201 \\
\hline & 239 & 221 & 460 & 206 & 242 & 448 \\
\hline & 110 & 118 & 228 & 135 & 133 & 268 \\
\hline & 115 & 115 & 230 & 180 & 200 & 380 \\
\hline & 117 & 112 & 229 & 135 & 164 & 299 \\
\hline & 118 & 126 & 244 & 127 & 136 & 263 \\
\hline 10 & 103 & 113 & 216 & 150 & 181 & 331 \\
\hline 1 & 98 & 100 & 198 & 130 & 164 & 294 \\
\hline 12 & 101 & 101 & 202 & 185 & 174 & 359 \\
\hline 13 & 95 & 105 & 200 & 105 & 137 & 242 \\
\hline 1 & 80 & 94 & 174 & 111 & 127 & 238 \\
\hline $6|-| 12$ & 937 & 984 & 1.921 & 1.258 & 1.416 & 2.674 \\
\hline $15-15$ & 181 & 158 & 339 & 213 & 294 & 507 \\
\hline Total & 1.357 & 1.363 & 2.720 & 1.677 & 1.952 & 3.629 \\
\hline
\end{tabular}

No último levantamento só foram examinadas as crianças e adolescentes que viveram sempre em Barretos. Consideraram-se como residentes contínuos todos os que durante suas vidas se ausentaram da cidade, no máximo trinta dias por ano, seguindo-se o critério estabelecido por Dean e col. ${ }^{5}$ (1935).

Nota-se que o número de examinados de 3 a 5 anos é similar nos dois levan- 
VIEGAS, Y. \& VIEGAS, A.R. Análise dos dados de prevalência de cárie dental na cidade de Barretos, SP, Brasil, depois de dez anos de fluoretação da água de abastecimento público. Rev. Saúde públ., S. Paulo, 19:287-99, 1985.

tamentos. A partir de 6 até 19 anos observa-se que o número examinado em 1981 foi aumentado em torno de $30 \%$.

\section{RESULTADOS E DISCUSSÃO}

Na Tabela 2 observa-se que o percen- tual de crianças de 3 a 5 anos de idade com dentes cariados apresentou uma redução da ordem de $59,7 \%$, e o percentual de redução correspondente a dentes extraídos foi equivalente a $70,4 \%$.

Se houve redução nesses dois percentuais, ocorreu, pelo contrário, aumento

TABELA 2

Percentagem de crianças de 3 a 5 anos de idade, segundo o sexo, com dentes cariados, obturados, extraídos e hígidos, na cidade de Barretos, SP, nos anos de 1971 e 1981.

\begin{tabular}{|c|c|c|c|c|c|c|c|c|}
\hline \multirow{3}{*}{ Sexo } & \multicolumn{8}{|c|}{ Dentes } \\
\hline & \multicolumn{2}{|c|}{ Cariados } & \multicolumn{2}{|c|}{ Obturados } & \multicolumn{2}{|c|}{ Extraídos . } & \multicolumn{2}{|c|}{ Hígidos } \\
\hline & 1971 & 1981 & 1971 & 1981 & 1971 & 1981 & 1971 & 1981 \\
\hline Masc. & 83,4 & 36,9 & 9,5 & 17,0 & 9,6 & 0,5 & 16,3 & 50,0 \\
\hline Fem. & 85,1 & 31,4 & 9,0 & 16,5 & 9,9 & 4,9 & 14,9 & 52,9 \\
\hline Total & 84,1 & 33,9 & 9,1 & 16,7 & 9,8 & 2,9 & 15,6 & 51,6 \\
\hline $\begin{array}{l}\text { Percen- } \\
\text { tual de } \\
\text { Diferença } \\
1971-1981\end{array}$ & \multicolumn{2}{|c|}{$-59,7$} & \multicolumn{2}{|c|}{$+83,5$} & \multicolumn{2}{|c|}{$-70,4$} & \multicolumn{2}{|c|}{$+230,7$} \\
\hline
\end{tabular}

nos percentuais de dentes restaurados $(83,5 \%)$ e de dentes hígidos $(230,7 \%)$.

Pelas Tabelas 3 e 4 pode-se verificar como ocorreu o comportamento do ata- que de cárie em relação ao número de dentes cariados e extraídos, respectivamente na dentição primária.

TABELA 3

Percentagem de crianças de 3 a 5 anos de idade, segundo o sexo, com zero, 1 a 4 e 5 e mais dentes cariados, na cidade de Barretos, SP, nos anos de 1971 e 1981.

\begin{tabular}{|c|c|c|c|c|c|c|}
\hline \multirow{3}{*}{ Sexo } & \multicolumn{6}{|c|}{ Número de dentes cariados } \\
\hline & \multicolumn{2}{|c|}{ Zero } & \multicolumn{2}{|c|}{$1|-| 4$} & \multicolumn{2}{|c|}{$5 \mathrm{e}+$} \\
\hline & 1971 & 1981 & 1971 & 1981 & 1971 & 1981 \\
\hline Masc. & 7,1 & 62,6 & 33,9 & 29,6 & 59,0 & 7,8 \\
\hline Fem. & 6,8 & 67,3 & 30,3 & 27,3 & 62,9 & 5,4 \\
\hline Total & 7,0 & 65,2 & 32,1 & 28,3 & 60,9 & 6,5 \\
\hline $\begin{array}{c}\text { Percentual de } \\
\text { Diferença } \\
1971-1981\end{array}$ & \multicolumn{2}{|c|}{$+831,4$} & \multicolumn{2}{|c|}{$-11,8$} & \multicolumn{2}{|c|}{$-836,9$} \\
\hline
\end{tabular}


VIEGAS, Y. \& VIEGAS, A.R. Análise dos dados de prevalência de cárie dental na cidade de Barretos, SP, Brasil, depois de dez anos de fluoretação da água de abastecimento público. Rev. Saúde públ., S. Paulo, 19:287-99, 1985.

TABELA 4

Percentagem de crianças de 3 a 5 anos de idade, segundo o sexo, com zero, 1 a 4 e 5 e mais dentes extraídos, na cidade de Barretos, SP, nos anos de 1971 e 1981.

\begin{tabular}{|c|c|c|c|c|c|c|}
\hline \multirow{3}{*}{ Sexo } & \multicolumn{6}{|c|}{ Número de dentes extraídos } \\
\hline & \multicolumn{2}{|c|}{ Zero } & \multicolumn{2}{|c|}{$1|-| 4$} & \multicolumn{2}{|c|}{$5 \mathrm{e}+$} \\
\hline & 1971 & 1981 & 1971 & 1981 & 1971 & 1981 \\
\hline Masc. & 87,5 & 95,6 & 12,1 & 2,9 & 0,4 & 1,5 \\
\hline Fem. & 90,0 & 94,6 & 9,5 & 5,4 & 0,5 & 0,0 \\
\hline Total & 88,8 & 95,1 & 10,8 & 4,2 & 0,4 & 0,7 \\
\hline $\begin{array}{l}\text { Percentual de } \\
\text { Diferença } \\
\text { 1971-1981 }\end{array}$ & \multicolumn{2}{|c|}{$+7,1$} & \multicolumn{2}{|c|}{$-61,1$} & \multicolumn{2}{|c|}{$+75,0$} \\
\hline
\end{tabular}

Os resultados desses dois tipos de dados apresentam um comportamento similar, assim é que se pode constatar que houve um aumento percentual das crianças com zero dente cariado e com zero dente extraído. Como decorrência desse evento observa-se uma redução na categoria de 1 a 4, tanto para os dentes cariados e extraídos, bem como na de 5 e mais dentes cariados. Apenas na categoria 5 e mais dentes extraídos é que não ocorreu esse fato, ocorrência esta que acreditamos seja casual, pois constitui uma exceção ao que foi observado como padrão nas demais categorias.

$\mathrm{Na}$ tabela 5 pode-se comparar o percentual de crianças sem cárie na dentição primária, observado após 10 anos de fluoretação, com o ocorrido em estudos com mesma duração no Brasil (Campi-

TABELA 5

Percentual de crianças sem cárie nos dentes primários, nas cidades de Barretos, SP, Campinas, SP, Grand Rapids (USA) e Holyhead (UK), após 10 anos de fluoretação da água.

\begin{tabular}{lcccc}
\hline & \multicolumn{4}{c}{ Cid a d e s } \\
\cline { 2 - 5 } $\begin{array}{c}\text { Grupo } \\
\text { etário }\end{array}$ & Barretos, SP & Campinas, SP* & $\begin{array}{c}\text { Grand Rapids } \\
\text { (USA)** }\end{array}$ & $\begin{array}{c}\text { Holyhead } \\
(\text { UK)** }\end{array}$ \\
\cline { 2 - 5 } & 48 & 47 & 51 & 54 \\
$31-15$ & 52 & $\ldots$ & $\ldots$ & 59 \\
\hline
\end{tabular}

* Viegas e Viegas ${ }^{2}(1974)$.

** Davies4 (1974). 
VIEGAS, Y. \& VIEGAS, A.R. Análise dos dados de prevalência de cárie dental na cidade de Barretos, SP, Brasil, depois de dez anos de fluoretação da água de abastecimento público. Rev. Saúde públ., S. Paulo, 19:287-99, 1985.

nas, SP), nos Estados Unidos (Grand Rapids) e no Reino Unido (Holyhead).

Pode-se concluir, pelos valores dos percentuais de crianças sem cárie, que os benefícios decorrentes da fluoretação em relação aos dentes da primeira dentição são semelhantes, nessas cidades, e que o benefício observado em Barretos é equivalente ao ocorrido nesses estudos. $\mathrm{Na}$ revisão de 55 estudos, Murray e Rugg-Gunn ${ }^{6}$ (1979) verificaram que $62 \%$ dos mesmos apresentaram reduções de cárie de 40 a $60 \%$ na dentição primária.

$\mathrm{Na}$ Tabela 6 pode-se observar o percentual de diferença relativo aos dentes permanentes cariados, obturados e extraídos, nas crianças de 7 a 10 e 7 a 14 anos. Nota-se que os percentuais seguem o mesmo padrão dos da dentição primária, tendo havido, pois, uma redução em relação aos dentes cariados e extraídos e um aumento nos percentuais de obturados e hígidos.

\section{TABELA 6}

Percentual de diferença em crianças de ambos os sexos, segundo grupos etários, dentes permanentes cariados, obturados, extraídos e hígidos, no período de 1971 a 1981 , na cidade de Barretos, SP.

\begin{tabular}{ccccc}
\hline \multirow{2}{*}{$\begin{array}{c}\text { Grupo } \\
\text { etário }\end{array}$} & \multicolumn{4}{c}{ D e n t e s } \\
\cline { 2 - 5 } & Cariados & Obturados & Extraídos & Hígidos \\
\hline $7-10$ & $-74,3$ & $+84,8$ & $-51,6$ & $+331,2$ \\
$7-14$ & $-67,9$ & $+29,5$ & $-55,8$ & $+340,7$ \\
\hline
\end{tabular}

Nas Tabelas 7 e 8 pode-se verificar como ocorreu o comportamento do ataque de cárie em relação ao número de dentes cariados e extraídos na dentição permanente.

Os resultados dos dois tipos de da-

TABELA 7

Percentual de diferença de ambos os sexos, segundo grupos etários, com zero, 1 a 4 e 5 e mais dentes cariados, no período de 1971 a 1981 , na cidade de Barretos, SP.

\begin{tabular}{cccc}
\hline \multirow{2}{*}{ Grupo etário } & \multicolumn{3}{c}{ Número de dentes cariados } \\
\cline { 2 - 4 } & Zero & $1|-| 4$ & 5 e mais \\
\hline $7|-| 10$ & +318 & $-62,1$ & -100 \\
$7-\mid 14$ & +226 & $-46,6$ & -89 \\
\hline
\end{tabular}

TABELA 8

Percentual de diferença em crianças de ambos os sexos, segundo grupos etários, com zero, 1 a 4 e 5 e mais dentes permanentes extraídos no período de 1971 a 1981, na cidade de Barretos, SP.

\begin{tabular}{cccc}
\hline \multirow{2}{*}{ Grupo etário } & \multicolumn{3}{c}{ Número de dentes extraídos } \\
\cline { 2 - 4 } & Zero & $1 \mid-4$ & 5 e mais \\
\hline $7|-| 14$ & $+2,5$ & $-38,8$ & 0,0 \\
$7 \mid 14$ & $+9,9$ & $-48,0$ & $-91,5$ \\
\hline
\end{tabular}


VIEGAS, Y. \& VIEGAS, A.R. Análise dos dados de prevalência de cárie dental na cidade de Barretos, SP, Brasil, depois de dez anos de fluoretação da água de abastecimento público. Rev. Saúde públ., S. Paulo, 19:287-99, 1985.

dos apresentam um comportamento similar; assim é que se pode constatar que houve uma redução nas categorias 5 e mais, 1 a 4 e, concomitantemente, houve aumento percentual de crianças com zero dentes cariados ou extraídos.

$\mathrm{Na}$ Tabela 9 observa-se o CPO médio por idade detectado nos anos de 1971 e
1981, podendo-se notar que houve uma redução em todas as idades. As reduções nas idades de 6 a 12 anos estão acima de $50 \%$, decrescendo nas de 13 e 14 anos, pois as crianças só começaram a receber $o$ benefício do flúor quando já tinham 3 e 4 anos de idade.

TABELA 9

CPO médio e percentagens de reduçāo em ambos os sexos, segundo a idade, observados no período de 1971 a 1981 , na cidade de Barretos, SP.

\begin{tabular}{|c|c|c|c|}
\hline \multirow{2}{*}{ Idade } & \multicolumn{2}{|c|}{ A no } & \multirow{2}{*}{ Percentual de Redução } \\
\hline & 1971 & 1981 & \\
\hline 6 & 1,51 & 0,55 & 63,6 \\
\hline 7 & 2,43 & 0,99 & 59,3 \\
\hline 8 & 3,10 & 1,52 & 51,0 \\
\hline 9 & 3,85 & 1,84 & 52,2 \\
\hline 10 & 5,09 & 2,22 & 56,4 \\
\hline 11 & 7,01 & 2,96 & 57,8 \\
\hline 12 & 8,37 & 3,82 & 54,5 \\
\hline 13 & 9,32 & 6,95 & 36,2 \\
\hline 14 & 10,54 & 8,81 & 16,4 \\
\hline
\end{tabular}

No grupo etário de 6 a 10 anos, que recebeu flúor durante toda sua vida, a redução foi da ordem de $55,4 \%$. Esta redução é equivalente à observada na maioria dos estudos de fluoretação. Murray e Rugg-Gunn ${ }^{8}$ (1979), ao avaliarem a eficiência da fluoretação, verificaram que em $62 \%$ dos estudos de fluo- retação da água, as reduções observadas situaram-se entre 50 e $70 \%$.

A Tabela 10 apresenta os dados referentes à percentagem de primeiros molares inferiores direitos atacados pela cárie. Nela pode-se observar que no grupo etário de 6 a 10 anos a redução foi da ordem de $43,4 \%$.

TABELA 10

Percentagem de crianças de ambos os sexos, com primeiro molar inferior direito (MID) atacado pela cárie, segundo a idade, observada no período de 1971 a 1981, na cidade de Barretos, SP.

\begin{tabular}{|c|c|c|c|}
\hline \multirow{2}{*}{ Idade } & \multicolumn{2}{|c|}{ A no } & \multirow{2}{*}{ Percentual de Redução } \\
\hline & 1971 & 1981 & \\
\hline 6 & 54,7 & 19,4 & 64,5 \\
\hline 7 & 66,8 & 31,0 & 53,6 \\
\hline 8 & 74,0 & 45,5 & 38,5 \\
\hline 9 & 76,9 & 49,8 & 35,2 \\
\hline 10 & 85,2 & 56,8 & 33,3 \\
\hline 11 & 82,8 & 63,9 & 22,8 \\
\hline 12 & 86,1 & 67,4 & 21,7 \\
\hline 13 & 92,0 & 79,3 & 13,8 \\
\hline 14 & 93,7 & 82,3 & 12,2 \\
\hline
\end{tabular}


VIEGAS, Y. \& VIEGAS, A.R. Análise dos dados de prevalência de cárie dental na cidade de Barretos, SP, Brasil, depois de dez anos de fluoretação da água de abastecimento público. Rev. Saúde públ., S. Paulo, 19:287-99, 1985.

Na Tabela 11 compara-se o percentual de crianças de 6 a 8 anos com primeiros molares permanentes sem cárie, como o ocorrido no Canadá, em Brantford e Stratford ${ }^{3}$, e nos EUA, em Newburgh².
Os valores de Barretos e Newburgh são similares, contudo inferiores aos de Brantford. Acreditamos que essa diferença ocorra por que o critério de dente cariado, seguido nos levantamentos, ao se examinar as fissuras e fossetas inclui,

TABELA 11

Percentagem de crianças de 6 a 8 anos de idade, de ambos os sexos, com primeiros molares permanentes sem cárie, nas cidades de Barretos, SP, Brantford, Canadá, Stratford, Canadá e Newburgh, USA.

\begin{tabular}{ccccc}
\hline \multirow{2}{*}{ Grupo etário } & \multicolumn{4}{c}{ C i d a d e s } \\
\cline { 2 - 5 } & Barretos & Brantford* & Stratford* & Newburgh** \\
\hline $6|-| 8$ & 50,9 & 76,53 & 75,83 & 52,9 \\
\hline
\end{tabular}

* Browin et al. ${ }^{3}$ (1954).

** Ast et al.2, cujo estudo refere-se a crianças de 6 a 9 anos.

além da condição do explorador prender, também a presença de tecido dentário amolecido ou mudança de coloração. Esse critério não é o seguido pelos clínicos quando examinam os dentes a fim de detectar se há ou não presença de cárie. Isso deve ocorrer provavelmente no Brasil e nos EUA com a maioria dos dentistas clínicos e daí verificar-se uma menor percentagem de primeiros molares permanentes sem cárie do que a observada no Canadá, onde os clínicos devem seguir o mesmo critério usado em saúde pública, que é o correto, a fim de não se restaurar dentes que não o necessitem.

$\mathrm{Na}$ cidade de Barretos, ao supervisionar o levantamento de 1981, um dos autores deste trabalho verificou, numa escola, que o número de MID atacados pela cárie (cariados, extraídos ou restaurados) era muito alto. Preocupado, pois nesse levantamento o CPO médio seria estimado por um de seus índices que inclui o ataque desse dente, perguntou ao examinador, que por coincidência era o cirurgião-dentista daquela escola, porque praticamente todos os MID estavam restaurados na face oclusal. Ele explicou que quando examinava os dentes das crianças inscritas no programa da escola a fim de verificar se a fissura apresentava ou não cárie, toda vez que o explorador prendesse, ele o considerava cariado e assim o restaurava. Disse ainda que esse critério ele tinha aprendido quando estudante.

Esse erro de diagnóstico, acreditamos, deve ser cometido pela maioria dos dentistas clínicos e, como consequiência, ao serem feitos os levantamentos observa-se uma redução significativamente menor do que a que deveria se observar em relação aos primeiros molares. Daí, crermos também que o conceito corrente de que o flúor protege menos as fissuras não seja tão exato quanto vem sendo apregoado, pois inúmeros desses dentes devem estar sendo restaurados sem que devessem sê-lo.

$\mathrm{Na}$ Tabela 12 pode-se verificar que os incisivos centrais superiores são altamente beneficiados pela fluoretação, sendo o percentual de redução nas crianças de 7 a 10 anos da ordem de $82 \%$. 
VIEGAS, Y. \& VIEGAS, A.R. Análise dos dados de prevalência de cárie dental na cidade de Barretos, SP, Brasil, depois de dez anos de fluoretação da água de abastecimento público. Rev. Saúde públ., S. Paulo, 19:287-99, 1985.

TABELA 12

Percentagem de crianças de ambos os sexos com incisivos centrais superiores atacados pela cárie, segundo a idade, observadas no período de 1971 a 1981 , na cidade de Barretos, SP.

\begin{tabular}{|c|c|c|c|}
\hline \multirow{2}{*}{ Idade } & \multicolumn{2}{|c|}{ A n o } & \multirow{2}{*}{ Percentual de Redução } \\
\hline & 1971 & 1981 & \\
\hline 7 & 4,4 & 0,8 & 81,8 \\
\hline 8 & 7,4 & 0,3 & 95,9 \\
\hline 9 & 17,2 & 4.2 & 75,6 \\
\hline 10 & 24,5 & 4,3 & 82,4 \\
\hline 11 & 35,9 & 6,4 & 82,2 \\
\hline 12 & 43,1 & 10,0 & 76,8 \\
\hline 13 & 49,5 & 21,1 & 57,4 \\
\hline 14 & 56,9 & 22,7 & 60,1 \\
\hline
\end{tabular}

Pela Tabela 13, pode-se constatar que no grupo etário de 7 a 10 anos, $50 \%$ das crianças, e no de 7 a $14,36 \%$ das crianças se apresentam isentas de cárie na dentição permanente.

TABELA 13

Percentual de crianças com dentes permanentes sem cárie, nas cidades de Barretos, SP, Campinas, SP e Grand Rapids, USA, após 10 anos de fluoração da água.

\begin{tabular}{cccc}
\hline \multirow{2}{*}{ Grupo etário } & \multicolumn{4}{c}{ Cid a de s } \\
\cline { 2 - 4 } & Barretos & Campinas* & Grand Rapids** \\
\hline $7|-| 10$ & 50 & 43 & 44 \\
$7|-| 14$ & 36 & 30 & 28 \\
\hline
\end{tabular}

* Viegas e Viegas ${ }^{9}(1974)$.

** Davies ${ }^{4}$ (1974) - 9 anos de fluoração.

Ao se analisar o percentual de re- Barretos, com Campinas ${ }^{9}$, Newburgh ${ }^{2}$, dução da cárie dental nos dentes per- Grand Rapids e Baixo Guandu ${ }^{7}$ (Tabemanentes das crianças da cidade de la 14), nota-se que são apresentados, nos

TABELA 14

Comparação de percentual de redução de cárie dental em dentes permanentes das crianças da cidade de Barretos, SP, Campinas, SP, Newburgh, USA, Grand Rapids, USA e Baixo Guandu, ES, após 10 anos de fluoração.

\begin{tabular}{rcccccc}
\hline \multirow{2}{*}{ Grupo etário } & \multicolumn{5}{c}{ Ci d a d e s } \\
\cline { 2 - 6 } & Barretos & Campinas* & Newburgh** & Grand Rapids*** & Baixo Guandu**** \\
\hline 6 & 55 & 63 & 58 & 61 & 69 \\
7 & -10 & 55 & 58 & $\cdots$ & 56 & 64 \\
10 & -12 & 56 & 50 & 53 & 53 & 58 \\
\hline
\end{tabular}

* Viegas e Viegas $^{9}(1974)$.

** Ast e col.2 (1958).

*** Davies 4 (1974)

**** Viegas 7 (1964). 
VIEGAS, Y. \& VIEGAS, A.R. Análise dos dados de prevalência de cárie dental na cidade de Barretos, SP, Brasil, depois de dez anos de fluoretação da água de abastecimento público. Rev. Saúde públ., S. Paulo, 19:287-99, 1985.

grupos etários comparados, valores similares aos observados nos outros estudos, com exceção dos do Baixo Guandu, que são maiores. Este fato pode ser explicado possivelmente porque o estudo do Baixo Guandu?, realizado pela Fundação SESP, assegurava, com maior segurança, que os examinados haviam realmente vivido na cidade, e por serem, nos levantamentos, na sua maioria, os mesmos examinadores.

E fato conhecido que os dentes já irrompidos também são beneficiados pela fluoretação. Essa a razão pela qual foram incluídos nos levantamentos de 1981 os indivíduos de 15 a 19 anos de idade. Assim é que no estudo de NewburghKingston de Ast e col. ${ }^{2}$ (1958), após 10 anos de fluoretação, os adolescentes de 16 anos de idade da cidade de Newburgh (fluoretada) apresentavam, na categoria de 15 ou mais dentes CPO, uma percentagem igual a $18 \%$, ao passo que as de Kingston ${ }^{2}$ (controle) o valor correspondente era igual a $63 \%$, o que equivale a uma redução de $71 \%$. Em Grand
Rapids, Arnold e col.1 (1956) observaram após 10 anos de fluoretação uma redução de cárie da ordem de $26 \%$ em crianças de 16 anos.

Nas Tabelas 15 e 16 pode-se observar como ocorreu o comportamento do ataque de cárie, em relação ao número de dentes cariados e extraídos no grupo etário de 15 a 19 anos. Os resultados dos dois tipos de dados apresentam o mesmo padrão, assim é que quanto aos dentes cariados houve uma redução na categoria 5 e mais, tendo ocorrido um aumento nas categorias 1 a 4 e zero.

Quanto à percentagem de dentes extraídos, observa-se uma redução evidente nas categorias 11 e mais e 5 a 10 $(-100,0 \%$ e $-46,2 \%)$, sendo que nas de 1 a 4 e zero, ocorreu um aumento, em ambas $(9,9$ e $0,6 \%)$.

Pelo comportamento dessas percentagens de cariados e extraídos, é lícito concluir que, sem dúvida, os dentes já irrompidos também são beneficiados pela fluoretação.

TABELA 15

Percentagem de adultos jovens de 15 a 19 anos de idade, segundo o sexo, com zero, 1 a 4 e 5 e mais dentes cariados, na cidade de Barretos, SP, nos anos de 1971 e 1981.

\begin{tabular}{lcccc}
\hline \multirow{2}{*}{ Sexo } & Ano & \multicolumn{3}{c}{ Número de dentes cariados } \\
\cline { 3 - 5 } & & Zero & $1-14$ & 5 e mais \\
\hline \multirow{2}{*}{ Masculino } & 1971 & 32,6 & 46,4 & 20,9 \\
& 1981 & 29,6 & 51,6 & 18,8 \\
Feminino & 1971 & 36,0 & 48,7 & 15,2 \\
& 1981 & 41,8 & 46,3 & 11,9 \\
\hline \multirow{2}{*}{ Total } & 1971 & 34,2 & 47,4 & 18,3 \\
& 1981 & 36,7 & 48,5 & 14,8 \\
\hline $\begin{array}{c}\text { Redução } \\
\text { 1971-1981 }\end{array}$ & & & & $-19,1$ \\
& & $+6,8$ & $+2,3$ & \\
\hline
\end{tabular}


VIEGAS, Y. \& VIEGAS, A.R. Análise dos dados de prevalência de cárie dental na cidade de Barretos, SP, Brasil, depois de dez anos de fluoretação da água de abastecimento público. Rev. Saúde públ., S. Paulo, 19:287-99, 1985.

\section{TABELA 16}

Percentagem de adultos jovens de 15 a 19 anos de idade, segundo o sexo, com zero, 1 a 4 , 5 a 10 e 11 e mais dentes extraídos, na cidade de Barretos, SP, nos anos de 1971 e 1981.

\begin{tabular}{|c|c|c|c|c|c|}
\hline \multirow{2}{*}{ Sexo } & \multirow{2}{*}{ Ano } & \multicolumn{4}{|c|}{ Número de dentes extraídos } \\
\hline & & Zero & $1-4$ & $5 \mid-10$ & 11 e mais \\
\hline \multirow[t]{2}{*}{ Masculino } & 1971 & 47,6 & 43,6 & 4,9 & 3,9 \\
\hline & 1981 & 47,5 & 48,9 & 3,6 & 0,0 \\
\hline \multirow[t]{2}{*}{ Feminino } & 1971 & 48,1 & 43,5 & 8,4 & 0,0 \\
\hline & 1981 & 48,6 & 48,0 & 3,4 & 0,0 \\
\hline \multirow[t]{2}{*}{ Total } & 1971 & 47,8 & 43,6 & 6,5 & 2,1 \\
\hline & 1981 & 48,1 & 48,4 & 3,5 & 0,0 \\
\hline $\begin{array}{l}\text { Percentual de } \\
\text { Redução } \\
\text { 1971-1981 }\end{array}$ & & $+0,6$ & $+9,9$ & $-46,2$ & $-100,0$ \\
\hline
\end{tabular}

Pela Tabela 17, verifica-se que ocor- percentagem de indivíduos que necessireu uma redução da ordem de $100 \%$ na tam dentaduras.

TABELA 17

Percentagem de adultos jovens de 15 a 19 anos de idade, segundo o sexo, com dentaduras, na cidade de Barretos, SP, nos anos de 1971 e 1981.

\begin{tabular}{lcccc}
\hline \multirow{2}{*}{ Sexo } & Ano & \multicolumn{3}{c}{ Dent a d u r a s } \\
\cline { 3 - 5 } & & Superior & Inferior & Ambas \\
\hline \multirow{2}{*}{ Masculino } & 1971 & 2,8 & 0,0 & 0,0 \\
& 1981 & 0,0 & 0,0 & 0,0 \\
Feminino & 1971 & 0,0 & 0,0 & 0,0 \\
& 1981 & 0,0 & 0,0 & 0,0 \\
\hline \multirow{2}{*}{ Total } & 1971 & 1,5 & 0,0 & 0,0 \\
& 1981 & 0,0 & 0,0 & 0,0 \\
\hline \multirow{2}{*}{$\begin{array}{c}\text { Percentual de } \\
\text { Redução } \\
1971-1981\end{array}$} & & & & - \\
\hline
\end{tabular}


VIEGAS, Y. \& VIEGAS, A.R. Análise dos dados de prevalência de cárie dental na cidade de Barretos, SP, Brasil, depois de dez anos de fluoretação da água de abastecimento público. Rev. Saúde públ., S. Paulo, 19:287-99, 1985.

\section{CONCLUSŌES}

Pela análise dos dados de dez anos de fluoretação da água de abastecimento da cidade de Barretos, pode-se concluir que:

1. As reduções observadas na prevalência de cárie, para os dentes primários e permanentes, são similares às constatadas em outros estudos, quer no Brasil ou no Exterior.

2. No grupo etário de 3 a 5 anos:

- $52 \%$ das crianças estão isentas 'de cárie.

- Houve um aumento na percentagem de crianças com dentes hígidos $(230,7 \%)$ e restaurados $(+83,5 \%)$, e uma redução nas com dentes extraídos $(-70,4 \%)$ e cariados $(-59,7 \%)$.

- O número de dentes cariados e extraídos apresentou na categoria 5 e mais reduções de 836,9 e $75 \%$; na categoria de 1 a 4, reduções de 11,8 e $61,1 \%$; e na categoria zero, aumentos de 831,4 e $7,1 \%$.

3. No grupo etário de 6 a 14 anos:

- Houve aumento na percentagem de crianças com dentes hígidos e restaurados, e redução nas com dentes cariados e extraídos.

- O número de dentes cariados e extraídos apresentou reduções nas categorias 5 e mais e 1 a 4 , e aumento na categoria zero.

- Houve redução no CPO médio em todas as idades. Nas idades de 6 a 10 fói observada redução de $55 \%$.

- Houve redução de $43 \%$ nas percentagem de primeiros molares permanentes atacados pela cárie no grupo etário de 6 a 10 anos.

- Houve redução de $82 \%$ na percentagem de incisivos centrais superiores, atacados pela cárie, no grupo etário de 7 a 10 anos.

4. No grupo etário de 15 a 19 anos:

- Os dentes cariados apresentaram redução percentual na categoria 5 e mais, e aumento nas categorias 1 a 4 e zero.

- Os dentes extraídos apresentaram reduções percentuais nas categorias 5 a 10 , e 11 e mais, e aumento nas categorias 1 a 4 e zero.

- Houve redução de $100 \%$ nas necessidades de dentaduras.

VIEGAS, Y. \& VIEGAS, A.R. [Data analysis on the prevalence of dental caries in Barretos city (S. Paulo, Brazil) after ten years of water fluoridation]. Rev. Saúde públ., $S$. Paulo, 19 :287-99, 1985.

ABSTRACT: Data of baseline and ten-year surveys of the prevalence of dental caries in children 3 to 14 years old, and young adults of 14 to 19 , in Barretos (S. Paulo State, Brazil), were analysed to verify the reduction observed in caries in the primary and permanent teeth during the ten-year period of water fluoridation. The reduction in the prevalence of dental caries observed is similar to the ones obtained in the pilot studies in Brazil and other countries. In the age group 7 to $10,50 \%$ of children do not have any permanent teeth attacked by caries, and $51.6 \%$ in the group 3 to 5 are in the same condition as regards their primary teeth.

UNITERMS: Fluoridation. Dental caries, prevention and control. Dental caries prevalence. 
VIEGAS, Y. \& VIEGAS, A.R. Análise dos dados de prevalência de cárie dental na cidade de Barretos, SP, Brasil, depois de dez anos de fluoretação da água de abastecimento público. Rev. Saúde públ., S. Paulo, 19:287-99, 1985.

\section{REFERENCIAS BIBLIOGRÁFICAS}

1. ARNOLD Jr., F.A. et al. Tenth year of the Grand Rapids-Muskegon study. Effect of fluoridated public water supplies on dental caries prevalence. $\mathrm{Publ}$. Hlth Rep., 71:652-8, 1956.

2. AST, D.B. et al. Newburgh-Kingston caries fluorine study. XV. Further observations of dental caries experience among children in Newburgh and Kingston, ages 7.16 based on DMF frequency per child. New York J. Dent., 28:173-5, 1958.

3. BROWIN, H.K, et al. Brantford fluoridation caries study: 1954 report. Canad. dent. Ass., 20:585-602, 1954.

4. DAVIES, G.N. Cost and benefit of fluorine in the prevention of dental caries. Geneva, WHO, 1974.

5. DEAN, H.T. et al. Mottled enamel in Texas. Publ. Hlth Rep., 50:424-42, 1935.

6. MURRAY, J.J. \& RUGG-GUNN, A.J. A review of the effectiveness of artificial water fluoridation throughout the world. [Apresentado em ORCA XXVI Congress, Scotland 1979 - Abstract n. ${ }^{\circ}$ 65]

7. VIEGAS, A.R. Cárie dental. Prevenção e tratamento em odontologia sanitária. Arq. Hig. Saúde públ., 29(102):189-206. 1964.

8. VIEGAS, A.R. Simplified indices for estimating the prevalence of dental cariesexperience in children seven to twelve years of age. J. publ. Hlth Dent., 29: $76-91,1969$

9. VIEGAS, Y. \& VIEGAS, A.R. Análise dos dados de prevalência de cárie dental na cidade de Campinas, SP, Brasil, depois de dez anos de fluoração da água de abastecimento público. Rev. Saúde públ., S. Paulo, 8:399-409, 1974.

Recebido para publicaçāo em 13/07/1982

Reapresentado em 17/04/1985

Aprovado para publicação em 09/05/1985 